\title{
A radiation oncology approach for using definitive radiotherapy with volumetric modulated arc therapy (VMAT) for skin field cancerisation (SFC)
}

\begin{abstract}
Patients with long histories of chronic ultraviolet radiation exposure can suffer from skin field cancerisation (SFC). They can have poor oncological outcomes and quality of life (QoL) with itch, flaking skin, and suboptimal cosmesis. Current treatments have high rates of treatment failure and non-compliance.

Definitive radiotherapy (RT) with volumetric modulated arc therapy (VMAT) has been proposed as a possible treatment modality in this clinical setting. This article provides a theoretical background to assist radiation oncologists (ROs) to implement this technique in their skin cancer practice.

The article covers the importance of the relationship between the skin physician and the RO, how to best understand the patient with SFC, patient assessment, preparation for RT, and RT planning and treatment.

The article also highlights the difficulty in measuring toxicities with the currently available tools and suggests future research options for affected patients. Clinical studies to further refine the use of RT in patients with SFC are warranted.

Keywords: skin neoplasms, radiotherapy, actinic keratosis, bowen's disease, in-situ squamous cell carcinoma, basal cell carcinoma, volumetric modulated arc therapy (VMAT), review
\end{abstract}

Volume 5 Issue 4 - 2018

\author{
Gerald B Fogarty,' David RH Christie, ${ }^{2}$ Art \\ Kaminski, ${ }^{3}$ Andrew E Potter ${ }^{4}$ \\ 'GenesisCare, St Vincent's Clinic, Australia \\ ${ }^{2}$ GenesisCare, Tugun, Australia \\ ${ }^{3}$ GenesisCare, Wesley Medical Centre, Australia \\ ${ }^{4}$ GenesisCare, St Andrew's Hospital, Australia
}

Correspondence: Gerald B Fogarty, GenesisCare, Department of Radiation Oncology, Mater Hospital, P.O. Box 1003, Crows Nest NSW 2065,Australia, Email gerald.fogarty@cancer.com.au

Received: May 07, 2018 | Published: July 16, 2018

\section{Introduction}

The incidence of skin cancer increases with age and its prevalence in the community is also on the rise. ${ }^{1}$ Patients with long histories of chronic ultraviolet radiation exposure can be affected by skin field cancerisation (SFC). These fields harbour pre-invasive cancer, especially actinic keratoses (AK), Bowen's disease and intraepithelial cancer, from which new invasive cutaneous squamous cell carcinoma (cSCC) can arise. $^{2}$ Affected patients can have poor oncological outcomes and years of poor quality of life $(\mathrm{QoL})$, with itch, flaking skin and suboptimal cosmesis.

Treatment of field cancerisation can be challenging utilising existing methods. Comorbidities often preclude certain modalities, especially extensive surgical excision which requires more than just primary closure or grafting. Patients may also decline surgery due to fear of poor functional or cosmetic outcomes, or even due to fatigue from repeat surgery. The durability of traditional non-surgical skin field treatments is disappointing. Recurrence within a short time frame is common. ${ }^{3-5}$

Definitive radiotherapy (RT) with volumetric modulated arc therapy (VMAT) has been proposed for SFC. ${ }^{6}$ VMAT is especially suited for extended skin field cancerisation (ESFC), defined as SFC over fifty square centimetres (cms) in area. ${ }^{6} \mathrm{ESFC}$ is a clinical scenario that deviates from the setting of isolated lesions more commonly encountered in a radiation oncology practice.

This article aims to assist radiation oncologists (ROs) to implement a VMAT skin cancer practice by outlining the theoretical background for this technique and highlighting how to approach patients and referrer relationships.

\section{Importance of the skin physician-radiation oncologist relationship}

Skin cancer patients are usually referred from a skin physician-a dermatologist, plastic surgeon, or general practitioner with an interest in skin cancer. It is common for the initial referral for radiotherapy to take place when other therapeutic options for the patient have been exhausted.

Prompt review and communication of the consultation outcome, preferably in real time by phone, is generally appreciated by the referrer. The provision of clear verbal answers that are subsequently supported by a written reply lend themselves in establishing longterm working relationships.

A working relationship between the skin physician and RO exists when both provide timely reciprocal services. The skin physician provides ongoing patient care through regular reviews which, with the use of dermatoscopy and biopsy, may facilitate the diagnosis of new problems. The RO provides RT when needed with prompt referral back to the skin physician once any acute RT effects have resolved. To ensure that the RO is aware of any late RT effects resulting from the relatively new VMAT skin technique, a collaborative research skin registry between the $\mathrm{RO}$ and skin physician has been created in Australia.

\section{Understanding the patient with SFC}

Patients with SFC constitute a distinct cohort of the case-mix that is conventionally managed in a radiation oncology practice. Their condition is usually chronic and managed over a long time course. 
Conventional treatment strategies may have included multiple surgical encounters and repeat topical applications. Commonly, these avenues may have been exhausted by the time of referral for RT. RT may be the only curative modality left for such patients, and to decline treatment may be resigning these individuals to decades of poor oncological outcomes and quality of life.

The mortality associated with skin cancer is likely to be lower when compared to other malignancies managed by radiation oncology departments, assuming other co-morbidities are accounted for. As such, it is imperative to be mindful of late RT effects, including the rare chance of secondary malignancy, ${ }^{8}$ as well as the potential need for future RT, which may impact on matching RT fields.

It is common for these patients to have more than one area involved by SFC. It may be possible to treat more than one field in a dedicated course of RT, bearing in mind the potential for toxicity. This may be instrumental in establishing the relative radiosensitivity of the patient and their condition.

\section{Patient assessment}

\section{History}

The history should outline the location of SFC and its duration. The dates and sites of prior treatments such as surgery, topical agents, and radiotherapy need to be elucidated.

Common topical treatments that may impact on local radiosensitivity include topical 5-fluorouracil-based creams $\left(\mathrm{Carac}^{\circledR}\right.$, Efudex $^{\circledR}$, Fluoroplex $\left.{ }^{\circledR}\right)$; trichloroacetic acid; the combination of diclofenac and hyaluronic acid $\left(\right.$ Solaraze $\left.^{\circledR}\right)$; imiquimod (Aldara ${ }^{\circledR}$, Zyclara ${ }^{\circledR}$ ); and ingenol mebutate $\left(\right.$ Picato $\left.^{\circledR}\right)$. Photodynamic therapy (PDT) also requires consideration. It is a local superficial therapy involving a two-step treatment process. A topical photosensitiser that is preferentially absorbed and accumulated in neoplastic tissue is applied first. Illumination with a specific light source then leads to the release of reactive oxygen species within the target tissue. Neoplastic cells are then destroyed by either necrosis or apoptosis. PDT is often administered in two fractions.

A history of sun exposure is the most common risk factor for developing cutaneous malignancies. Another common risk factor for developing cutaneous malignancies is immune suppression. Immune suppression may be present due to an underlying condition, including a number of autoimmune disorders or chronic lymphocytic leukaemia (CLL). Immune suppression may be conferred by an acquired condition such infection with the Human Immunodeficiency Virus (HIV). Immune suppression may also be a consequence of medical treatment of a number of medical conditions with agents such as corticosteroids, azathioprine or methotrexate for benign conditions, or cytotoxic agents used to manage malignant conditions. A special case of immune suppression is found in a cohort of patients who have undergone solid organ or bone marrow transplantation, with the requisite ensuing immunosuppressant therapy.

Immune suppression may not only predispose to skin cancer development, but also can impact on the tolerability of radiotherapy and hence ability to deliver a definitive course of treatment. Furthermore, a compromised blood supply may also impact the tolerability of radiotherapy. Conditions associated with vascular compromise include peripheral vascular disease, diabetes, and a number of autoimmune conditions, in particular the vasculitidies.
Occupational exposure to sensitising agents may include chemical carcinogens, including arsenic and inorganic arsenic compounds, or polycyclic aromatic hydrocarbons in tars. ${ }^{9}$ Family history for syndromes associated with skin malignancy in the young, such as basal cell nevus syndrome (BCNS), also need to be considered. This syndrome is caused by pathogenic variants in PTCH1 and PTCH2, and is associated with an increased risk of basal cell carcimona (BCC). Syndromes such as xeroderma pigmentosum (XP), oculocutaneous albinism, epidermolysis bullosa, and Fanconi anaemia are associated with an increased risk of cutaneous squamous cell carcinoma (cSCC).

Active symptoms and their impact on activities of daily living, including sleep should be recorded. The presence of formication may be indicative of perineural invasion by tumour. Associated masses may be suggestive of spread to local lymph nodes. A history of worsening symptoms in the summer months is common due to the skin being re-challenged by ultra violet radiation (UVR).

\section{Examination}

Examination will usually reveal an older Caucasian person with histological proof of keratinocytic lesions in the area in question. Physical examination requires a thorough skin check under adequately lit conditions. Areas of SFC and ESFC need to be elucidated and those for RT marked out. Sometimes these areas will cross natural boundaries as shown in the Figure 1 example of the shin and dorsum of foot. These areas can still be treated with VMAT. Large convex surfaces of skin field cancerisation are usually in sun exposed areas and include the scalp, forehead, cheeks, forearms, legs, chest, upper back and shoulders.

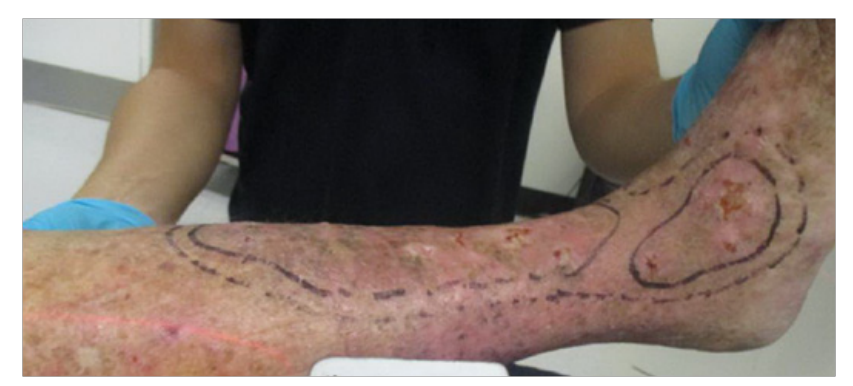

Figure I Shin and dorsum of foot involved in ESFC-the volume to be treated crosses the ankle joint.

The patient should be asked which areas are most symptomatic. This creates a triage list of fields to be treated if there is more than one. Sometimes, it is best to treat fields sequentially rather than synchronously. For example, an aged patient living alone may have their ability to self-care threatened if they have too many areas treated at once. This is because acute RT effects can limit mobility with pain and swelling. Having large areas of skin in the acute phase of radiation effects may also impact their homeostasis and maintain body temperature.

Scars from previous surgeries need to be identified in the field being considered for RT as the patient may not remember where they are located. Previous surgery, especially those involving more complicated closures, such as flaps and grafts, can drive in-situ disease deeper which will affect the radiation target volume depth. Surgery can also disrupt lymphatics and slow the resolution of acute radiation effects, especially in dependent limbs. This may necessitate 
slower fractionation or a treatment break. Treatment in the vicinity of a skin graft needs to be deferred by at least four weeks from the time of surgery in order to permit adequate healing.

It is important to determine the depth of in-situ change. The recognised depth of the epidermis is $4.5 \mathrm{~mm},{ }^{10}$ but in advanced SFC it can be more. Tumour bulk is better appreciated by palpation rather that inspection alone. The skin can be thickened with intraepidermal cancerisation, and this will affect the depth needing treatment. When possible, bimanual palpation (nares, ears, lips, cheeks, webs of fingers) can help confirm this. Some thickening may be due to scarring or lymphatic obstruction due to prior therapy. Any existing lymphoedema, especially in association with previous scars, needs to be noted as it can flare during radiotherapy.

Staging is also important. The field in question needs to be thoroughly examined to see if there are any active invasive lesions that may require a higher dose or another therapy (e.g. surgical excision or curettage) prior to the commencement of radiotherapy. Higher dose delivery is possible using a simultaneous integrated boost (SIB) approach. ${ }^{11}$ Draining lymph node fields need palpating, and signs of perineural invasion need to be excluded. Investigations will be driven by the history and examination. The presence of high risk features may warrant imaging to exclude metastatic disease. Biopsy evidence of malignancy needs to be documented.

\section{Preparation for radiotherapy}

Patients referred, selected and assessed for RT need to have clear explanation of what to expect from treatment in order to provide informed consent. The RO needs to explain the rationale, process and side-effects, both acute and late. Consent needs to cover the possibility of mucositis, wet desquamation and lymphoedema, especially in a dependent limb. As VMAT will deliver doses from unique beam angles, side-effects in organs in the entrance and exit paths need to be considered, with particular attention paid to surrounding organs at risk (OARs) such as the lacrimal and salivary glands when treating the head and neck region.

Treatment of the lower part of the face may warrant a dental review prior to RT planning. Patients with any part of the digestive tract in the radiation volume should also be considered for review with a dietitian. An ophthalmology review prior to RT is warranted if any part of the orbit is anticipated being in the treatment volume. Review with a lymphoedema specialist should be sought for those who have previously undergone lymphadenectomy, especially if there has been surgery to the groin or axilla.

A routine radiation nursing assessment should be carried out where the focus is on skin care, the possible need for dressings, and how these will be organised. The need for topical dressings should be anticipated towards the end of treatment and for an ensuing period thereafter. The importance of adequate nutrition and hydration during treatment needs to be impressed upon patients. Maintaining weight is also essential to avoid variation in contour and separation during RT. $\mathrm{RT}$ is planned assuming that the separation will be constant during the course of treatment.

Transport assistance, especially towards the end of therapy when acute RT toxicity will be expected, needs to be ascertained to ensure treatment continuation and completion.

\section{RT Planning}

\section{Simulation}

After informed consent has been obtained, a planning appointment is scheduled. At this appointment the RO confirms the field(s) to be treated, as previously discussed with the patient and referring doctor. These are then marked on the patient's skin using a bright light and erasable marker. The maximum dimension of any area to be treated with a modern linear accelerator is 40 centimetres $(\mathrm{cms})$ at the isocenter. Some other technologies, such as TomoTherapy ${ }^{\circledR}$, may be able to treat longer fields.

Areas within the treatment volume requiring a higher dose for macroscopic disease should be marked to assist the creation of a SIB volume at the contouring stage. The skin markings should outline the clinical target volume (CTV) ${ }^{12}$ which is then contoured in the treatment planning system.

Clinically invasive lesions within the field need to be individually marked as gross tumour volumes (GTVs) ${ }^{12}$ numbered sequentially, and noted in preparation for SIB. A convention is to number from proximal to distal, and from medial to lateral, but any system is acceptable as long as it is consistent.

When marking lesions of the head, neck and chest, a handheld mirror is useful so that the patient can also visualise the area(s) being targeted for treatment. In the case of limbs, the RO will need to make a decision whether or not to cover all the disease. Total coverage may compromise the ability to spare a corridor of skin to preserve dermal lymphatic drainage, which may impact on lymphoedema risk. Further research is needed to see if this is necessary given that VMAT can spare deep central lymphatics.

The areas drawn on the patient are copied onto plastic sheets as templates ${ }^{11}$ which are then stored electronically in the patient's file. This record allows fields to be reproduced in the future to determine if disease recurrence is in-field, at field edge, or out of field. Saving this information also assists future salvage therapy, if required.

The patient is immobilised ready for scanning according to department protocol which may involve masks, body shells and vacuum bags. Patient positioning must allow for bolus placement to ensure that the full dose is delivered to the skin surface. Bolus is most stable when applied horizontally and firmly taped to the limb being treated. Three-dimensional bolus printing solutions are being developed to ensure reproducibility and reduce treatment times.

Careful attention to patient positioning reduces unnecessary irradiation of other body parts. For example, when treating a lower limb that will be extended for RT, the other limb is flexed at the hip and knee to avoid dose from the entrance and exit beams. If this is not possible, the legs can be separated to maximise the arc length used to treat around the leg (Figure 2). For arms, the patient can adopt the "Superman" position (Figure 3), which involves lying prone on the treatment couch with the arm being treated extended above the head. The other (untreated) arm may be positioned by the patient's side to avoid beam contact. In this position, forearms are treated in pronation which is the optimal position relative to where the SFC is usually located. The usual field is then located on the superior aspect of the arm and is more easily visualised for treatment set up. Bolus can then also be applied and taped with the advantage of gravity. 


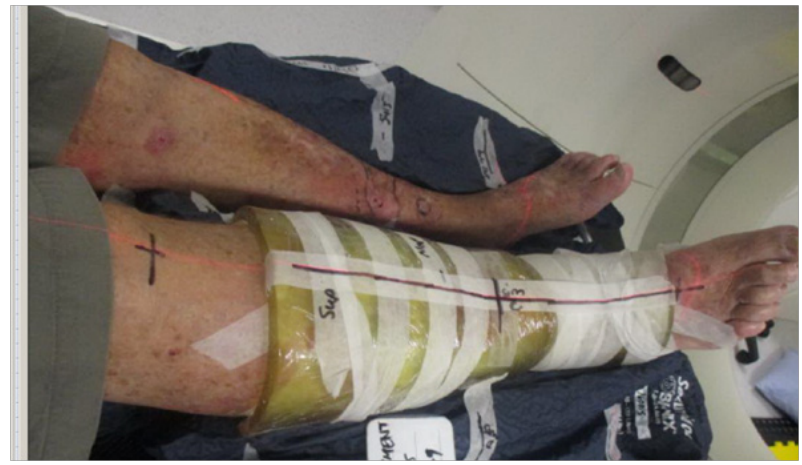

Figure 2 Positioning needs to ensure that other body parts will not be in the treatment arcs. The right leg is elevated to maximize the angle that the arc can treat around it so that the left leg will not get in the way. Bolus wrapped around the area to be treated enables the full dose to be deposited on the skin.

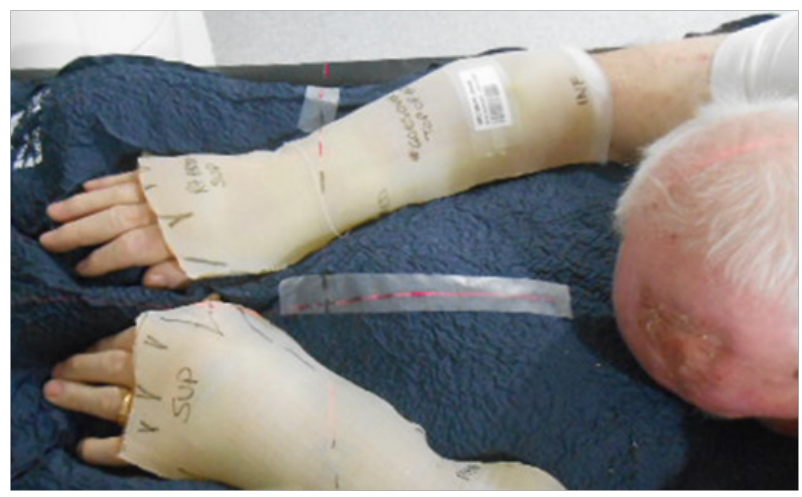

Figure 3 Positioning the patient in the 'superman' position removes the body out of the arc that the linear accelerator will use to irradiate both arms.

Immobilisation aids need to be considered. Head and neck fields require a personalised thermoplastic mask. Limbs require a vacuum bag, and treatment to the torso benefits from a body cradle. To ensure the RO marks are captured on the planning scan, wire is laid on the marks, or onto any immobilisation device covering the area, e.g. a mask. Wires need to be of sufficient diameter to be easily identified on the planning CT scan. The wires aid RO contouring on the scan at the planning stage. It is essential to planning success that any wire placement is precise, and that it is applied in such a way that it does not move during the scanning process.

Wires can also be placed around OARs or areas where dose should be minimised. For example, wiring the eyebrows may assist in reducing dose to this region when planning a forehead treatment. Marks can also be put on the skin by the RO and physicist where in-vivo dosimetry can be measured to ensure that the correct dose is being delivered. All dosimetry points should be photographed on the patient at simulation and wired. For head and neck regions, the pen marks are transposed to the mask, and wire is then placed on the outer surface of the mask.

Build up or bolus material, usually of one-centimetre thickness, is used to ensure full dose with six megavoltage (6MV) photons to the skin. This energy is the usual energy used, but other energies are possible. Higher energies may require a thicker bolus, which may be less conformal on complex curved surfaces. Thicker bolus may slip during treatment, and can also be an uncomfortable weight for the patient to bear. The bolus needs to extend peripherally at least 2 $\mathrm{cm}$ beyond the wired area at the periphery of the field for dosimetric reasons. Bolus may be applied prior to scanning with as many airgaps removed as possible.

A planning computed tomography (CT) scan is performed to encompass the treatment area. The CT planning scan is done in the treatment position usually at $0.2 \mathrm{~cm}$ slice thickness. It is important to label the right and left sides at scanning as these scans are often done feet-to-gantry or prone, and the orientation may be different to a routine scan for an internal organ. Multiple areas may require multiple scans in different set up positions. Scanning needs to extend above and below the area of interest to contour the OARs which may include the complete lungs, lacrimal glands, hippocampi, etc.

The patient can be asked to try different manoeuvres during the scan that will assist in removing the OARs from the treatment volume. For example, looking away from the volume to be irradiated during beam on time removes the anterior chamber of the eyes from a forehead that is being treated. The patient is taken off the CT couch, has the marks removed, and will next be seen during treatment.

\section{Contouring}

The scan is imported into the treatment planning system (TPS). RO planning then takes place with the RO contouring the volumes to be treated on the TPS using the wires placed at simulation. There are several aids known to planners which can assist this. The RO contours the GTVs for SIB as aided by the wires placed at simulation. A similar process is followed to contour the CTV.

The treatment volumes for skin cancers are a product of the area and depth. Wires determine the area, and depth is determined by clinical examination. When contouring in-situ disease that is not thickened on palpation, the maximum depth of the skin appendages is $4.5 \mathrm{~mm},{ }^{10}$ so the CTV should be to a depth of $5 \mathrm{~mm}$.

Contouring the CTV can be assisted by creating a volume of skin minus $5 \mathrm{~mm}$ and then applying that result and the skin auto contour. Sometimes the skin is less than $5 \mathrm{~mm}$ deep e.g. in the scalp or pretibial region. An auto contour using the skull can help, assuming that invasive disease does not grossly invade the skull, which should have been clinically determined by physical examination. Interpolation must be checked, especially in the scalp to minimise dose to the brain.

The PTV is created by automatic expansion as per departmental protocol. The expansion will depend on the immobilisation and reliability of the reproducible set up. The PTV is expanded into the bolus to aid dosimetry and requires radiation therapist and physics input. Typical expansion is $3 \mathrm{~mm}$ with $5 \mathrm{~mm}$ into bolus. With a CTV of $5 \mathrm{~mm}$ this gives a total PTV thickness of $13 \mathrm{~mm}$.

The resultant treatment volumes are a rind around organs that need to be spared (Figure 4). OARs are contoured and auto contours are checked for veracity.

\section{Radiation dose prescription}

The RO then prescribes the radiation dose, with consideration of total dose, dose per fraction, dose per week, and the possibility of a treatment break. The total dose for in-situ cSCC is controversial. In a 2012 review of seven retrospective studies, Zygogianni et al., ${ }^{13}$ showed that the average dose was 40Gray (Gy) (range: 10-70Gy) in 10 to 20 fractions. A diverse number of modalities and techniques, 
including external beam, orthovoltage and megavoltage beams, brachytherapy moulds and unsealed sources of radioactive paint, were included. Some physicians prescribed high doses as per invasive disease, whereas others prescribed lower doses because of the noninvasive nature of in-situ cSCC, sensitive anatomic locations (e.g. an extremity), large treatment areas, or the desire to decrease the number of fractions in older patients.
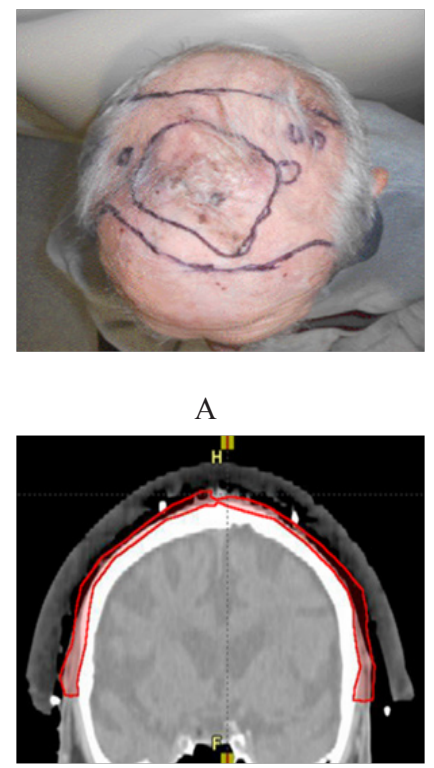

$\mathrm{B}$

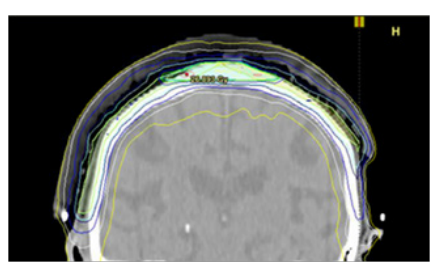

C

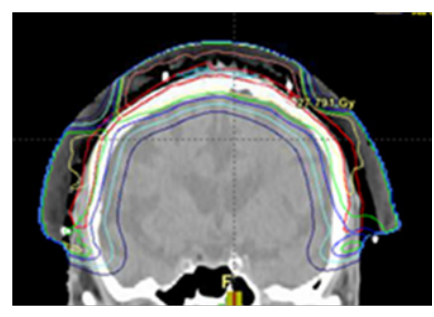

D

Figure 4 Photographs and CT plans of a patient preparing for VMAT.

A: Mark-up of a scalp field at planning showing areas of GTV within the PTV.

B: CTV is contoured on the planning scan as a rind around the brain being the OAR.

C:A sagittal CT planning image showing the resultant dosimetry with VMAT avoiding the brain. The dose homogeneity is uniform throughout the target. The dose profile across the field is uniform with no need for junctioning radiation fields.

D: In contrast, the same patient with an electron plan of the scalp with the necessary field junctions with inferior dosimetry. (Figures provided courtesy of Prof Peter Graham)

The dose per fraction in Zygogianni et al., ${ }^{13}$ varied from $2 \mathrm{~Gy}$ per day to $5 \mathrm{~Gy}$, the latter given at a maximum of twice weekly. The authors commented that all studies showed an increase in late skin toxicities, including skin necrosis, with daily doses above 4Gy.

The dose per week is controversial. Some practitioners argue that in-situ disease is susceptible to accelerated repopulation and that it should be treated without a break for fear or accelerated repopulation. ${ }^{14}$ Others feel that this is not the case, and that a break has no impact on the total dose. Other groups believe that a break is necessary to avoid unacceptable short-term toxicity such as pain and swelling. One practice is to mandate a two week treatment break between fraction 10 and 11 of a 25 -fraction course. No data is available regarding the radiobiological effect of treatment breaks when using VMAT for skin treatment, however this is under active investigation.

Our approach is to use a dose of $45 \mathrm{~Gy}$ in 25 fractions to in-situ disease with SIB to 55-60Gy for areas of invasive carcinoma. The dose prescription point is important. VMAT gives a uniform dose through the treatment volume and has none of the "top down" problems ${ }^{6}$ associated with fixed source external beam solutions or topical therapy. A "top down" problem is when there is excess dose at the top of the epidermis, resulting in toxicity, but insufficient dose at depth to cause permanent sterilisation of premalignant basal cells which can eventually lead to in-field recurrence.

Limbs with vascular problems will have difficulty in healing acute radiation toxicity, and standard fractionation with 2Gy per day, or even hyper fractionation with 1.5-1.8Gy per day, is the most prudent approach. Dose parameters needed for sterilisation of in-situ disease of other types of cancers may help to predict what dose is needed for in-situ cSCC. Clinical dose studies for in-situ cSCC are needed.

\section{Plan acceptance}

At plan acceptance, the RO will need to understand that significant dose may extend beyond the PTV, and the relevant OARs that may receive entry and exit dose will need to be contoured for assessment. The number of arcs required will depend on dose homogeneity and dose to OARs.

\section{Quality assurance (QA)}

As is usual with a VMAT plan, quality assurance needs to be undertaken. This ensures that the computer generated treatment plan can be delivered in a safe and practical manner. The timing of QA will depend on institutional protocol and workflow of the associated medical physicists.

\section{Treatment}

The removal of finger rings in the early treatment period may be warranted for patients receiving arm treatment, especially for those with risk factors for swelling e.g. patients who are dialysis dependent, have arteriovenous fistulas, or have undergone previous axillary lymphadenectomy.

On-treatment reviews (OTRs) should be undertaken at weekly intervals throughout treatment, with the option of increasing frequency according to the severity of skin reaction. Some patients can have reactions that are painful, and some react to RT early in the course of treatment, showing a tendency to radiosensitivity. A break, or even treatment cessation, may be needed. Some experimental protocols have a built-in break, usually after 10 fractions. The cause of the early reaction is not known. It may be underlying genetic radiation sensitivity ${ }^{15}$ or environmental causes such as previous therapies, concurrent medications, or comorbidities such as immunosuppression. 
Accelerated repopulation ${ }^{14}$ has not been reported as yet for VMAT for ESFC. Radio-biological investigation is needed.

\section{The difficulty of measuring acute skin toxicity in SFC with the current tools}

The current acute radiation gradings for $\operatorname{skin}^{16,17}$ are based on the skin being an OAR rather than the organ that requires treatment alone. Such grading scales have been predominantly used in breast radiotherapy. In this setting, the skin is usually a bystander organ. Significant effort in breast radiotherapy is expended on avoiding radiation to non-target skin to avoid acute and late effects. Breast techniques with the same oncological outcomes are compared in their ability to avoid skin side-effects. Megavoltage radiotherapy is used, especially for its skin sparing capability, and treatment is fractionated to minimise late effects. When skin is thought to be involved with cancer, bolus is used to deliver more dose to skin.

VMAT for SFC is considerably different to VMAT for other tumours. The skin is abnormal in that it has suffered from exposure to UVR and treatments, with these perhaps affecting radiation sensitivity. The skin also becomes the target tissue as the location of cancer to be eradicated. A comparison of the acute effects of radiotherapy in normal non-UVR damaged skin versus skin with ESFC reveals major differences. Comparison (Figure 5) (Figure 6) of images of normal skin versus ESFC shows reliance of acute effects on fractionation and the difference between normal and abnormal skin to treatment. Figure 5 is a case study of hypofractionated RT (45Gy in 15 fractions at 5 per week). The axilla skin is non-UVR damaged. Figure 6 contrasts the radiobiological sequence with VMAT treated UVR-damaged skin with ESFC using hyperfractionated RT (36Gy in 20 fractions at 5 per week). The latter case experienced significant wet desquamation at a lower dose, but this could be due to tumor lysis. Perhaps a separate grading system is needed.

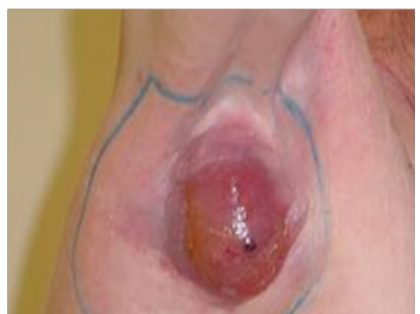

A

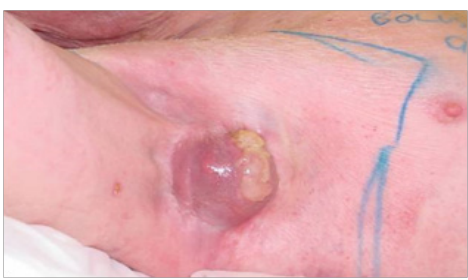

B

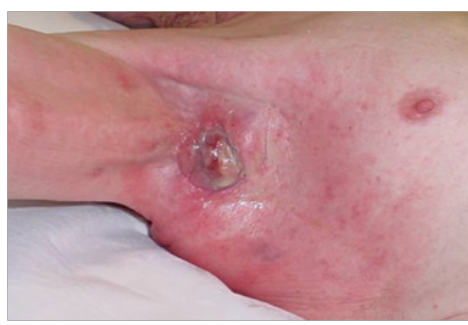

$\mathrm{C}$

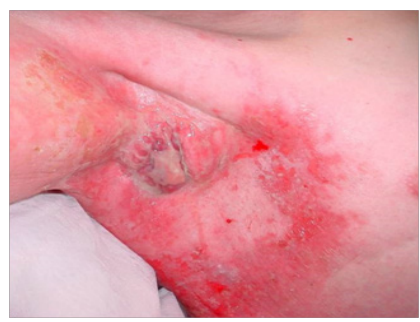

$\mathrm{D}$

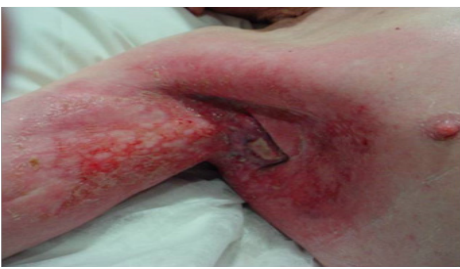

E

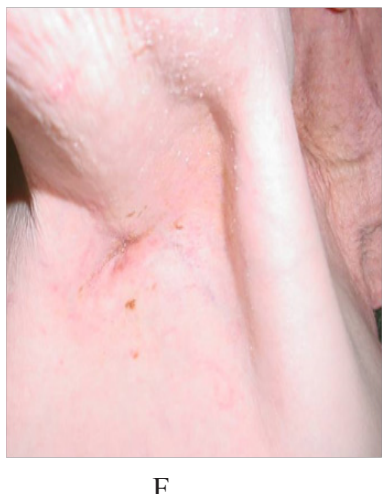

Figure 5 A case study to review the acute RT effects in normal skin, the reliance of the time course of RT effects on fractionation, and how this differs to cancer.The axilla skin is non-UVR damaged.

A: The axilla bears an inoperable lymph node that has been taken over by metastatic cSCC from a hand primary. Treatment consists of definitive RT alone. The dose prescribed is $45 \mathrm{~Gy}$ in 15 fractions at 5 per week $(45 / 15 / 5)$ with full dose to normal skin. Bolus has been placed on the skin to ensure delivery of the full dose to skin as this is from a skin primary.

B:At 27Gy (9 of I5 fractions, two weeks into RT). The RT field is outlined in blue chinagraph. There is no skin reaction yet. However, the tumour is suffering acute effects. The yellow material on the tumour is tumorlysis or necrosis, not pus.

C:At 7 days post RT, erythema is present in the normal skin within the field. D:At 14 days post RT, wet desquamation is present in field at the peak of the RT reaction. This is not cellulitis, as the reaction is only in-field. At this stage, there is a need for careful skin care as the skin is open. There is no need for antibiotics.

E:At 2 I days following the cessation of RT recovery is progressing with the formation of islands of new skin from surviving skin stem cells.

F: At 120 days the skin is fully healed and a complete response is observed for the cancer.

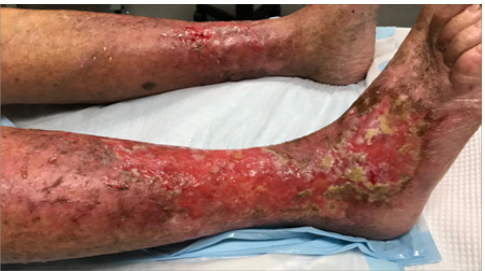

A

Citation: Fogarty GB, Christie DRH, Kaminski A, et al. A radiation oncology approach for using definitive radiotherapy with volumetric modulated arc therapy (VMAT) for skin field cancerisation (SFC). Int J Radiol Radiat Ther. 2018;5(4):227-234. DOI: 10.15406/ijrrt.2018.05.00168 


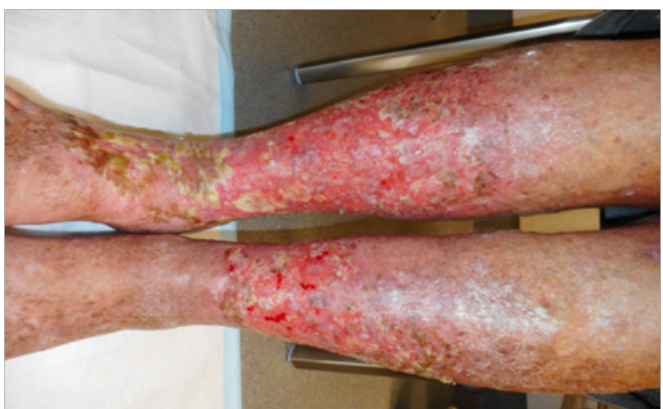

B

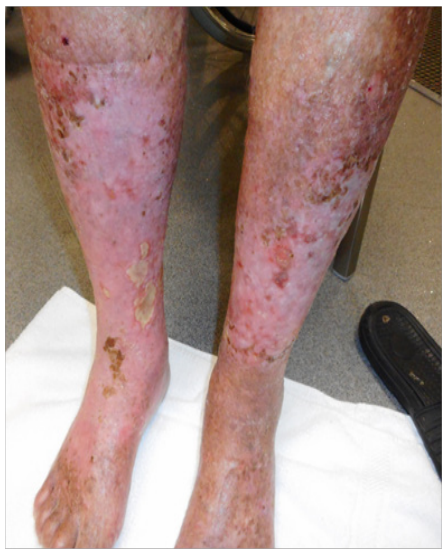

$\mathrm{C}$

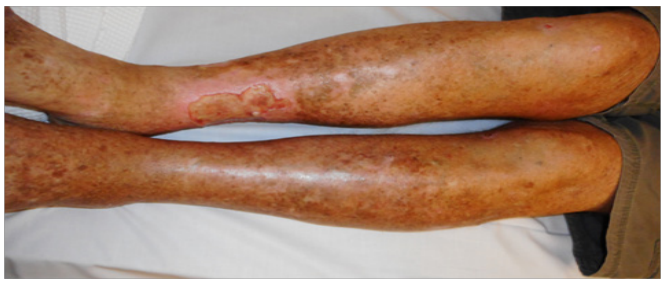

$\mathrm{D}$

Figure 6 The leg seen in Figure I after VMAT for ESFC. Both legs were treated.

A:At 3 days following $36 \mathrm{~Gy}$ in 20 fractions. The total prescribed dose of $54 \mathrm{~Gy}$ in 60 fractions at 5 per week $(54 / 60 / 5)$ was not given due to increasing pain in both legs. This reaction may be tumour lysis rather than true wet desquamation. In normal skin, wet desquamation is usually associated with doses of at least $40 \mathrm{~Gy}$ in 20 fractions.

B:At 7 days following 36/20. Pain has resolved and there is rapid reepithelialization.

C:At 12 days following 36/20. Re-epithelialization is almost complete.

D:At 75 days following 36/20. Re-epithelialization is complete. There is no ESFC or pain.

\section{Future research opportunities}

\section{Radiation Physics}

Improvements in dose distribution may be achieved by the use of novel techniques such as tangential VMAT. ${ }^{18}$

This is shown graphically in Figure 7.

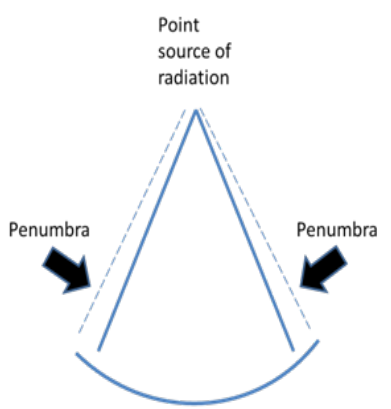

A

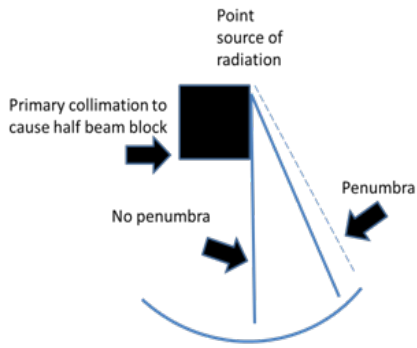

B

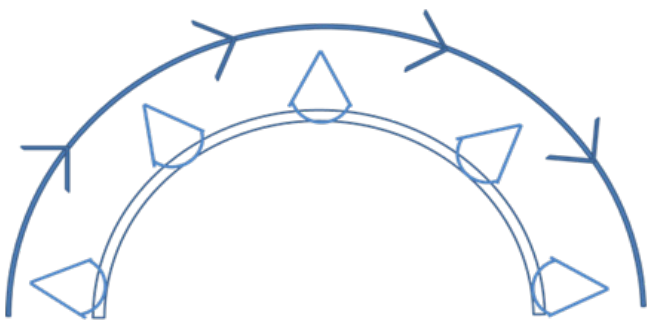

C

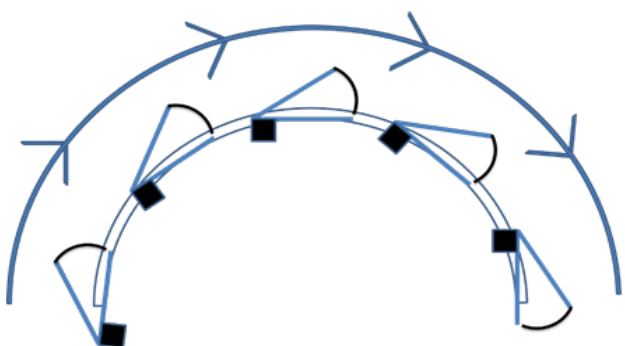

D

Figure 7 The theoretical basis of tangential VMAT.VMAT is essentially CT technology applied to intensity modulated radiotherapy (IMRT).

A: The penumbra of the beam can add unwanted dose. The penumbra is the dose at the edge of the beam that is not a full dose, so it cannot be regarded as therapeutic, but it is also not a zero dose, so it can add to the dose suffered by OARs.

B:The primary collimation of the beam can be used to decrease the width of the penumbra. This is known as "half beam blocking."

C: Current VMAT.The axis of the beam points towards the target. However, this means that there is transmission of dose through the PTV to the underlying OARs.

D: Tangential VMAT is when the axis of the beam is perpendicular to the convexity of the target. When combined with half beam blocking, this significantly decreases the dose transmitted through the PTV to underlying OARs. 


\section{Radiobiology}

The integration of RT with other modalities such as systemic or topical agents may improve the therapeutic ratio. A wide range of doses have been reported as effective in the treatment of in-situ cSCC, and further investigation of the dose-response relationship is warranted. The clinical effect of split-course treatment, and in particular the risk of accelerated repopulation, is also an area of ongoing interest.

\section{Conclusion}

Volumetric modulated arc therapy (VMAT) is a valid treatment option for skin field cancerisation. Skin physicians and patients are looking for radiation oncologists who can take this new skin cancer treatment technique forward. ${ }^{6}$ Radiation oncologists need a theoretical framework in order to approach this new therapy in a logical manner, which this article aims to provide. Phase I clinical trials to refine the physics and radiobiological aspects of treatment are planned in order to assess the safety and efficacy of VMAT in skin field cancerisation, with a view to a randomised trial of radiotherapy versus current best topical therapy.

\section{Acknowledgements}

The authors wish to thank Aileen Eiszele for editing assistance and manuscript preparation.

\section{Funding sources}

There are no funding sources to declare.

\section{Conflicts of interest}

The authors have no conflicts of interest to declare.

\section{References}

1. Staples MP, Elwood M, Burton RC, et al. Non-melanoma skin cancer in Australia: the 2002 national survey and trends since 1985. Med J Aust. 2006;184(1):6-10.

2. Green AC. Epidemiology of actinic keratoses. Curr Probl Dermatol. 2015;46:1-7.

3. Krawtchenko N, Roewert Huber J, Ulrich M, et al. A randomised study of topical 5\% imiquimod vs. topical 5-fluorouracil vs. cryosurgery in immunocompetent patients with actinic keratoses: a comparison of clinical and histological outcomes including 1-year follow- up. $\mathrm{Br} \mathrm{J}$ Dermatol. 2007;157(2):34-40.

4. Askew DA, Mickan SM, Soyer HP, et al. Effectiveness of 5-fluorouracil treatment for actinic keratosis-a systematic review of randomized controlled trials. Int J Dermatol. 2009;48(5):453-463.
5. Gupta AK, Paquet M, Villanueva E, et al. Interventions for actinic keratoses. Cochrane Database of Systematic Reviews. 2012;12.

6. Fogarty GB, Christie D, Spelman LJ, et al. Can modern radiotherapy be used for extensive skin field cancerisation: An update on current treatment options. Biomed J Sci \&Tech Res. 2018;4(1).

7. Spelman L. National dermatology radiation oncology registry: A human research registry to collect information from patients referred to radiation oncologists for the management of non-melanoma and melanoma skin cancer and difficult to manage inflammatory skin diseases through radiotherapy.

8. Fogarty GB, Shumack S. Common dermatology questions and answers about the radiation treatment of skin cancer in the modern era. Int $J$ Radiol Radiat Ther. 2018;5(2):108-114.

9. Diepgen TL, Drexler H. Occupational skin cancer. In: Rustemeyer T, Elsner P, editors. Kanerva's Occupational Dermatology. Springer; Berlin: 2012

10. Fogarty GB, Hong A, Scolyer RA, et al. Radiotherapy for lentigo maligna: a literature review and recommendations for treatment. $B r J$ Dermatol. 2014;170(1):52-58.

11. Martin TD, Moutrie Z, Tighe D, et al. Volumetric modulated arc therapy (VMAT) for skin field cancerisation of the nose $-\mathrm{A}$ technique and case report. Int J Radiol Radiat Ther. 2018;5(3):142-148.

12. Prescribing, recording and reporting photon beam therapy. International Commission on Radiation Units \& Measurements. Bethesda: 1993.

13. Zygogianni A, John K, Maria T, et al. The potential role of radiation therapy in Bowen's disease: a review of the current literature. Rev Recent Clin Trials. 2012;7(1):42-46.

14. Bese NS, Hendry J, Jeremic B. Effects of prolongation of overall treatment time due to unplanned interruptions during radiotherapy of different tumor sites and practical methods for compensation. Int $J$ Radiat Oncol Biol Phys. 2007;68(3):654-661.

15. Fogarty G, Muddle R, Sprung C, et al. Unexpectedly severe acute radiotherapy side effects are associated with single nucleotide polymorphisms of the melanocortin-1 receptor. Int J Radiat Oncol Biol Phys. 2010;77(5):1486-1492.

16. https://en.wikibooks.org/wiki/Radiation_Oncology/Toxicity_grading/ RTOG

17. https://www.eviq.org.au/clinical-resources/assessment-tools/231radiation-induced-skin-reaction-assessment-sca

18. Santos ED, Green JA, Bhandari N, et al. Tangential volumetric modulated radiotherapy-A new Technique for large scalp lesions with a case study in lentigo maligna. Int J Bioautomation. 2015;19(2):223-236. 